\title{
Diagnosis of cystic fibrosis related diabetes: a selective approach in performing the oral glucose tolerance test based on a combination of clinical and biochemical criteria
}

\author{
Bernard Yung, Michael Kemp, James Hooper, Margaret E Hodson
}

\begin{abstract}
Background-Cystic fibrosis related diabetes (CFRD) has become increasingly common with the increasing longevity of patients with cystic fibrosis. The diagnosis of CFRD is important as its development may lead to a clinical deterioration which may be reversed with treatment. The oral glucose tolerance test (OGTT) is the method of choice in the diagnosis of CFRD, but performing OGTTs on all patients is inconvenient for patients and labour intensive for staff. The aim of this study was to identify a more selective approach in performing OGTTs in the diagnosis of CFRD based on the use of a combination of clinical and biochemical criteria.
\end{abstract}

Methods-Clinically stable adult patients with cystic fibrosis not known to be diabetic attending the Royal Brompton Hospital Cystic Fibrosis Clinic for their annual review were invited to return within a month to have an OGTT. The result of the OGTT was compared with the results of tests performed during the annual review. The sensitivities and specificities of various methods used in the screening or diagnosis of CFRD were determined using OGTT as the "gold standard" diagnostic method. The combination of clinical and biochemical criteria which resulted in the highest sensitivity and specificity in the diagnosis of CFRD was determined.

Department of Cystic Fibrosis

B Yung

M E Hodson

Department of Clinical Biochemistry

M Kemp

J Hooper

Royal Brompton Hospital, London SW3 6NP, UK

Correspondence to: Dr B Yung, Flat 11, Marlin House, 22 St John's Avenue, East Putney, London SW15 2AA, UK.

Received 22 December 1997 Returned to authors 16 March 1998 Revised manuscript received 20 May 1998 Accepted for publication 26 August 1998
Results-Between August 1996 and May 1997122 patients became eligible for the study, 91 of whom agreed to take part. The number of patients with normal, impaired, and diabetic glucose tolerance was $58(64 \%), 21(23 \%)$, and $12(13 \%)$, respectively. When used alone, abnormal glycosylated haemoglobin (HbA1c) was found to have the highest sensitivity $(83 \%$; $95 \%$ CI 62 to 100) in the diagnosis of CFRD. The combination of an abnormal random blood glucose and/or abnormal HbA1c and/or symptoms of hyperglycaemia or weight loss was found to have the highest sensitivity (92\%; 95\% CI 76 to 100$)$ in the diagnosis of CFRD. The specificity of this combination in the diagnosis of CFRD was $79 \%$ (95\% CI 70 to 88$)$. By selectively performing OGTTs in patients with one or more of the criteria cited above, 11 of the 12 patients with OGTT defined diabetes would have been identified.
Conclusions-Patients with cystic fibrosis already have to undergo a large number of routine investigations. The selective approach in performing OGTTs described here has the potential to identify the majority of patients with CFRD without the need to perform this investigation on all patients. This approach is likely to be welcomed by patients and will lead to significant savings in terms of time and resources for patients and staff. Further larger studies are warranted to validate this selective approach in the diagnosis of CFRD.

(Thorax 1999;54:40-43)

Keywords: cystic fibrosis; diabetes mellitus; glucose tolerance test

Cystic fibrosis related diabetes (CFRD) is a well recognised complication in patients with cystic fibrosis and the prevalence of this complication increases with age. ${ }^{12}$ The diagnosis of CFRD is important as its development may be associated with clinical deterioration ${ }^{34}$ which can be reversed by prompt treatment. ${ }^{4}$ Various methods when used alone-including random blood glucose (RBG), fasting blood glucose (FBG), glycosylated haemoglobin (HbA1c), and symptoms of hyperglycaemiahave not been found to be sufficiently sensitive or specific in the diagnosis of CFRD. ${ }^{56} \mathrm{~A}$ two hour oral glucose tolerance test (OGTT) is generally regarded as the "gold standard" in the diagnosis of diabetes but performing OGTTs is time and resource consuming for both patients and staff. However, in the absence of any other standardised and practical diagnostic test, OGTT remains the method of choice in the diagnosis of CFRD. It has been suggested that all patients aged 10 or above with cystic fibrosis should have this test periodically. ${ }^{5}$ The aim of the study was to identify a more selective approach in performing OGTT in the diagnosis of CFRD, based on the use of a combination of clinical and biochemical criteria. The sensitivities and specificities of various clinical and biochemical criteria, when used alone, in the diagnosis of CFRD would also be determined using OGTT as the "gold standard".

\section{Methods}

Adult patients (aged 16 or above) with cystic fibrosis not known to be diabetic who attended the Royal Brompton Hospital Adult Cystic 
Table 1 Demographic details and results of the 91 patients who underwent OGTTs between August 1996 and May 1997

\begin{tabular}{|c|c|c|c|c|}
\hline & Total & $N G T$ & $I G T$ & $D G T$ \\
\hline No. of patients studied & 91 & $58(63.7 \%)$ & $21(23.1 \%)$ & $12(13.2 \%)$ \\
\hline$M: F$ & $58: 33$ & $40: 18$ & $12: 9$ & $6: 6$ \\
\hline $\mathrm{Age}^{\star}$ & $27(8)$ & $27(7)$ & $25(7)$ & $30(11)$ \\
\hline $\mathrm{BMI} \star$ & $21(2.9)$ & $21(2.9)$ & $20(3.1)$ & $20(3.1)$ \\
\hline Number of patients on long term oral steroids & 9 & 5 & 1 & 3 \\
\hline Number of patients on enteral feeding & 3 & 1 & 1 & 1 \\
\hline \multicolumn{5}{|l|}{ Number of patients with established chronic } \\
\hline liver disease (hepatomegaly) & 8 & 3 & 3 & 2 \\
\hline Pancreatic insufficient:pancreatic sufficient & $81: 10$ & $48: 10$ & $21: 0$ & $12: 0$ \\
\hline \multicolumn{5}{|l|}{ Results: } \\
\hline $\mathrm{RBG}(\mathrm{mmol} / \mathrm{l})^{\star}$ & $6.0(2.4)$ & $5.3(1.6)$ & $6.2(2.8)$ & $8.9(2.5)$ \\
\hline $\mathrm{HbA} 1 \mathrm{c}(\%)^{\star}$ & $5.8(1.2)$ & $5.6(1.1)$ & $5.8(1.1)$ & $7.0(1.1)$ \\
\hline \multicolumn{5}{|l|}{ Patients with symptoms of hyperglycaemia } \\
\hline and/or weight loss & 17 & 5 & 5 & 7 \\
\hline Patients with glycosuria & 4 & 1 & 1 & 2 \\
\hline $\mathrm{FBG}(\mathrm{mmol} / \mathrm{l})^{\star}$ & $5.2(1.1)$ & $4.9(0.6)$ & $5.2(0.7)$ & $6.9(1.8)$ \\
\hline Two hour glucose $(\mathrm{mmol} / \mathrm{l})^{\star}$ & $7.8(4.2)$ & $5.6(1.4)$ & $9.3(1.1)$ & $16.3(4.6)$ \\
\hline
\end{tabular}

NGT = normal glucose tolerance; $\mathrm{IGT}=$ impaired glucose tolerance; $\mathrm{DGT}=$ diabetic glucose tolerance BMI = body mass index; $\mathrm{RBG}=$ random blood glucose; HbA1c = glycosylated haemoglobin; FBG = fasting blood glucose .

${ }^{\star}$ Figures are given as mean (SD).

Fibrosis Clinic for their annual review between August 1996 and May 1997 were invited to take part in the study. Patients with pulmonary exacerbations requiring oral or intravenous antibiotic therapy, recent (within six weeks) increase or change in systemic steroid dosage, recent commencement of enteral feeding, and pregnant patients were excluded. Cystic fibrosis was diagnosed by positive sweat tests with typical clinical findings, with or without genotype confirmation.

Each eligible patient was invited to take part in this study during the annual review visit. For those patients who agreed to take part, the following information was collected: RBG (patients with $\mathrm{RBG}>11.0 \mathrm{mmol} / 1$ are regarded as diabetic by the WHO criteria ${ }^{7}$ ), HbAlc (normal $<6.2 \%$ ), the presence of symptoms of hyperglycaemia, unexplained weight loss $(>5 \%$ of weight loss since previous visit), the presence of glycosuria.

Each patient was then invited to return to the clinic within one month in order to have a two hour OGTT performed. After an overnight fast the patient was asked to drink a solution containing $1.75 \mathrm{~g} / \mathrm{kg}$ body weight (maximum $75 \mathrm{~g}$ ) of glucose BP dissolved in $250 \mathrm{ml}$ of water within 2-3 minutes. Blood samples were taken just before and two hours after ingestion of the glucose solution. Patients' glucose tolerance status was classified according to the WHO criteria into normal, impaired, or diabetic glucose tolerance (two hour venous plasma glucose $<7.8 \mathrm{mmol} / 1,7.8-11.0 \mathrm{mmol} / \mathrm{l}$, $>11.0 \mathrm{mmol} / \mathrm{l}$, respectively). ${ }^{7}$

The result of the OGTT was then compared with the result collected during the annual review visit. The sensitivity and specificity of

Table 2 Sensitivities and specificities of the use of individual clinical and biochemical criteria in the diagnosis of CFRD using the OGTT as the "gold standard" diagnostic method

\begin{tabular}{|c|c|c|}
\hline & $\begin{array}{l}\text { Sensitivity } \\
(95 \% C I)\end{array}$ & $\begin{array}{l}\text { Specificity } \\
(95 \% \text { CI })\end{array}$ \\
\hline Random blood glucose $(>11.0 \mathrm{mmol} / \mathrm{l})$ & $33 \%(7$ to 60$)$ & $97 \%(94$ to 100$)$ \\
\hline Glycosylated haemoglobin $>6.1 \%$ & $83 \%(62$ to 100$)$ & $89 \%(82$ to 96$)$ \\
\hline $\begin{array}{l}\text { Symptoms of hyperglycaemia and/or unexplained } \\
\text { weight loss }\end{array}$ & $58 \%(30$ to 86$)$ & $87 \%(80$ to 95$)$ \\
\hline Presence of glycosuria & $17 \%(0$ to 38$)$ & $97 \%(94$ to 100$)$ \\
\hline Fasting blood glucose $(>7.7 \mathrm{mmol} / \mathrm{l})$ & $25 \%(1$ to 50$)$ & $100 \%$ \\
\hline
\end{tabular}

each individual diagnostic method compared with the OGTT were calculated as follows:

Sensitivity of individual method in the diagnosis of $\mathrm{CFRD}=$

No. of patients with CFRD determined

by this method

True no. of patients with CFRD deter- $\times 100 \%$ mined by OGTT

Specificity of individual method in the diagnosis of $\mathrm{CFRD}=$

True no. of patients without CFRD determined by OGTT — no. of patients without CFRD determined by OGTT but with an abnormal result by the method used True no. of patients without CFRD deter- $\times 100 \%$ mined by OGTT

The same calculations were then made to determine the sensitivities and specificities of using various combinations of clinical and biochemical criteria described above in the diagnosis of CFRD. Three cut off values for RBG (6, 8.5 and $11 \mathrm{mmol} / \mathrm{l}$ ) were chosen. According to the WHO criteria, ${ }^{7}$ diabetes is "likely" in patients with RBG above $11 \mathrm{mmol} / 1$ and "unlikely" if RBG is $6 \mathrm{mmol} / 1$ or below; 8.5 $\mathrm{mmol} / \mathrm{l}$ represents the mid point of these two values. The combination of criteria which resulted in the highest sensitivity and specificity was then determined.

Blood samples for plasma glucose were collected in fluoride oxalate tubes and venous plasma glucose was determined by an oxygen rate method using a Beckman CX 7 Delta analyser (Beckman Instruments, USA). Blood samples for the determination of $\mathrm{HbAlc}$ were collected in EDTA-containing tubes and $\mathrm{HbAlc}$ was determined by an ion capture assay using an Abbott IMX analyser (Abbott Laboratories, USA). The presence of glycosuria was determined by Multistix (Bayer Diagnostics, UK).

The study was approved by the local ethics committee and written consents were obtained from all patients who participated.

\section{Results}

A total of 518 patients with cystic fibrosis of median age 26 (range 16-60) attended the Royal Brompton Hospital Cystic Fibrosis Clinic in August 1996 of whom 74 patients 
Table 3 Sensitivities and specificities (95\% CI) of the use of various clinical and biochemical criteria in the diagnosis of CFRD in the 91 patients using the OGTT as the "gold standard" diagnostic method

\begin{tabular}{|c|c|c|c|c|c|c|c|c|c|}
\hline $\begin{array}{l}\text { Clinical or biochemical } \\
\text { criteria used in the } \\
\text { selection }{ }^{\star} \text { of patients to } \\
\text { undergo OGTTs }\end{array}$ & $\begin{array}{l}H b A 1 c \\
>6.1 \%\end{array}$ & $\begin{array}{l}\text { Presence of } \\
\text { glycosuria }\end{array}$ & $\begin{array}{l}\text { Presence of } \\
\text { symptoms of } \\
\text { hyperglycaemia or } \\
\text { unexplained weight } \\
\text { loss }\end{array}$ & $\begin{array}{l}R B G \\
>11.0 \\
m m o l / l\end{array}$ & $\begin{array}{l}R B G \\
>8.5 \\
\mathrm{mmol} / \mathrm{l}\end{array}$ & $\begin{array}{l}R B G \\
>6.0 \\
\mathrm{mmol} / \mathrm{l}\end{array}$ & $\begin{array}{l}\text { Sensitivity } \\
(95 \% \text { CI) in the } \\
\text { diagnosis of CFRD }\end{array}$ & $\begin{array}{l}\text { Specificity } \\
(95 \% \text { CI) in the } \\
\text { diagnosis of CFRD }\end{array}$ & $\begin{array}{l}\text { Using a selective } \\
\text { approach, }{ }^{\star} \text { no. of OGTTS } \\
\text { that would have been } \\
\text { performed due to the } \\
\text { presence of one or more of } \\
\text { the criteria }\end{array}$ \\
\hline a & Yes & Yes & Yes & Yes & \multirow{3}{*}{ Yes } & \multirow{4}{*}{ Yes } & $92 \%(76$ to 100$)$ & $79 \%(70$ to 88$)$ & 28 \\
\hline $\mathrm{b}$ & Yes & Yes & Yes & & & & $92 \%$ (76 to 100$)$ & $74 \%$ (65 to 84$)$ & 31 \\
\hline c & Yes & Yes & Yes & & & & $92 \%(76$ to 100$)$ & $65 \%(54$ to 75$)$ & 39 \\
\hline$d^{\star \star}$ & Yes & & Yes & Yes & \multirow{3}{*}{ Yes } & & $92 \%$ (76 to 100$)$ & $79 \%(70$ to 88$)$ & 28 \\
\hline $\mathrm{e}$ & Yes & & Yes & & & \multirow{3}{*}{ Yes } & $92 \%$ (76 to 100$)$ & $75 \%(65$ to 84$)$ & 31 \\
\hline$f$ & Yes & & Yes & & & & $92 \%(76$ to 100$)$ & $65 \%(54$ to 75$)$ & 39 \\
\hline $\mathrm{g}$ & Yes & & & Yes & \multirow{3}{*}{ Yes } & & $83 \%$ (62 to 100 ) & $86 \%$ (78 to 94$)$ & 21 \\
\hline $\mathrm{h}$ & Yes & & & & & & $83 \%(62$ to 100$)$ & $84 \%$ (75 to 92$)$ & 23 \\
\hline $\mathrm{i}$ & Yes & & & & & Yes & $92 \%$ (76 to 100$)$ & $70 \%(59$ to 80$)$ & 35 \\
\hline
\end{tabular}

${ }^{\star}$ Selective approach $=$ performing OGTTs only in patients with one or more of the abnormal criteria (marked 'Yes') cited above.

$\star \star$ The presence of an abnormal RBG $(>11.0 \mathrm{mmol} / \mathrm{l})$ and/or an abnormal HbA1c $(>6.1 \%)$ and/or the presence of symptoms of hyperglycaemia or unexplained weight loss has the highest sensitivity and specificity in the diagnosis of CFRD. Including the presence of glycosuria did not increase these values further.

were known to be diabetic (prevalence 14\%). Between August 1996 and May 1997, 152 patients attended the Cystic Fibrosis Clinic for annual review. Thirty patients were not eligible to be included in this study including 23 patients who were known to have diabetes. Of the remaining 122 patients ( 80 male) who were eligible for the study, 91 agreed to participate. Inability to travel to the hospital in the morning due to work commitments or long distance to travel were the usual reasons for the patients' inability to take part in the study. The demographic details and the results of the OGTTs of the 91 patients studied is shown in table 1 . The mean age and body mass index were not significantly different between those who took part in the study and those who were unable to do so. The sensitivities and specificities of individual clinical and biochemical criteria alone in the diagnosis of CFRD, using OGTT as the "gold standard" diagnostic method for CFRD, is shown in table 2.

An abnormal $\mathrm{HbA} 1 \mathrm{c}$ was found to have the highest sensitivity $(83 \% ; 95 \%$ CI 62 to 100$)$ when used alone in the diagnosis of CFRD. By combining the use of different diagnostic criteria, the sensitivity in the diagnosis of CFRD could be increased further (table 3 ). The highest sensitivity achieved was $92 \%$ (95\% CI 76 to 100) in patients who had either abnormal RBG $(>11.0 \mathrm{mmol} / \mathrm{l})$ and/or abnormal HbA1c $(>6.1 \%)$ and/or the presence of symptoms of hyperglycaemia or unexplained weight loss. The presence of glycosuria did not increase the sensitivity further. This higher sensitivity was achieved at the expense of a lower specificity of $79 \%$ (95\% CI 70 to 88 ) so some patients with one or more of the abnormal criteria cited above may not have CFRD. By selectively performing OGTTs only in this group of patients with one or more of the abnormal criteria cited above, those patients within the group with true CFRD would have been identified and those without CFRD within the group would have been excluded. If such a selective approach was used for the 91 patients studied, only $28(31 \% ; 95 \%$ CI 21 to 41$)$ of the 91 patients would have to undergo an OGTT, but 11 of the 12 patients $(92 \%, 95 \%$ CI 76 to 100$)$ with OGTT defined diabetes within the group would have been identified. The twelfth patient had a normal RBG, HbAlc and was asympto- matic and would not have been identified unless an OGTT was performed.

\section{Discussion}

Various methods, including OGTT, have been used at different cystic fibrosis centres for the screening and diagnosis of CFRD. At the authors' unit patients with cystic fibrosis have been screened for diabetes at least annually using RBG and $\mathrm{HbA} 1 \mathrm{c}$ for some years. In this study we found that an abnormal RBG ( $>11.0$ $\mathrm{mmol} / \mathrm{l}$ ), when used alone, has a poor sensitivity in the diagnosis of CFRD. In common with the study by Lanng and colleagues, ${ }^{5}$ we also found that an abnormal FBG and the presence of symptoms of hyperglycaemia when used alone have poor sensitivity in the diagnosis of CFRD. Lanng and colleagues reported that only $16 \%$ of their patients with OGTT defined diabetes had abnormal HbA1c. This is in contrast to $83 \%$ (95\% CI 62 to 100$)$ of our diabetic patients with abnormal HbA1c values. In the Danish study, ion exchange chromatography was used to measure $\mathrm{HbAlc}$ and a higher reference range was used (upper limit $6.4 \%$ ). These differences are insufficient to explain the large differences in the sensitivities between the two studies. HbA1c is a specific type of glycated haemoglobin formed by the reaction of glucose with the $\mathrm{N}$-terminal amino group of the haemoglobin beta chain. It provides an integrated measure of past glucose levels and would be expected to be increased in patients with diabetes. ${ }^{8}$ We are unable to explain the low percentage of OGTT defined diabetics with abnormal HbA1c levels in the Danish cystic fibrosis patient group. Although the presence of glycosuria has a high specificity in the diagnosis of CFRD, this test is too insensitive to be used as a diagnostic or screening test for the condition. Our study therefore provides further evidence that methods other than OGTT, when used alone, are not sufficiently sensitive or specific in the diagnosis of CFRD. However, performing an OGTT on all patients is very inconvenient for the patients and labour intensive for staff. The need to have an OGTT performed regularly may in some cases decrease patients' compliance with clinic visits.

In this study we have shown that a selective approach in performing OGTTs has the 
potential to significantly reduce the total number of patients with cystic fibrosis who would have to undergo OGTTs yet would enable the majority of diabetic patients to be identified. As CFRD occurs almost exclusively in patients with pancreatic exocrine insufficiency, ${ }^{9}$ performing OGTTs in patients who are pancreatic sufficient may not be necessary, thus further reducing the number of OGTTs needed to be performed. The small proportion of patients with OGTT defined diabetes not identified by the selective approach described above would be those with normal RBG, HbA1c, and no symptoms of hyperglycaemia or unexplained weight loss. We speculate that the clinical and metabolic consequences of their diabetes may not be as great as those diabetics with one or more of the abnormal criteria cited above. As most adult patients are reviewed at least three monthly, these patients are likely to be identified at a later date.

Patients with cystic fibrosis already have to undergo a large number of regular investigations. A more selective approach in performing OGTTs in the diagnosis of CFRD described in this study is likely to be welcomed by patients. Further larger studies are warranted to validate this selective approach in the diagnosis of CFRD.

We gratefully acknowledge the patients who took part in this study, the staff of the Department of Clinical Biochemistry for performing the blood assays, Mrs J Turner for statistical advice, performing the blood assays, Mrs J Turner for statistical advice, Sister S Scott for performing some of the OGTTs,
sor D Geddes for allowing us to study his patients.

Funding: none.

Funding: none.

1 Dodge JA, Morrison G. Diabetes mellitus in cystic fibrosis: a review. F $R$ Soc Med 1992;85(Suppl 19):25-8.

2 Hodson ME Diabetes mellitus and cystic fibrosis. Bailliere' Clinical Endocrinology and Metabolism 1992;6:797-805.

3 Finkelstein SM, Wielinski CL, Elliott GR, et al. Diabetes Finkelstein SM, Wielinski CL, Elliott GR, et al. Diabetes
mellitus associated with cystic fibrosis. $\mathcal{F}$ Pediatr 1988;112: mellitus

4 Lanng S, Thorsteinsson B, Nerup J, et al. Diabetes mellitus in cystic fibrosis: effect of insulin therapy on lung function and infections. Acta Pediatr 1994;83:849-53.

5 Lanng S, Hansen A, Thorsteinsson B, et al. Glucose tolerance in patients with cystic fibrosis: five year prospective study. BMF 1995;311:655-9.

6 Yung B, Kemp M, Hooper J, et al. Random blood glucose alone in the diagnosis of cystic fibrosis-related diabetes. Lancet 1997;349:619.

7 WHO. Diabetes mellitus. Technical report series 727 . Geneva: WHO, 1985.

8 Svendsen PA, Christiansen JS, Soegaard U, et al. Rapid changes in chromatographically determined haemoglobin A1c induced by short-term changes in glucose concentraAlc induced by short-term changes
tion. Diabetologia 1980;19:130-6.

9 Geffner M E, Lippe B M, Kaplan S A, et al. Carbohydrate tolerance in cystic fibrosis is closely linked to pancreatic exocrine function. Pediatr Res 1984;18:1107-11. 